\title{
Tracking Phagosome-Derived Vesicles in Macrophages with Microfabricated Microparticles
}

\author{
Wenhao Cheng ${ }^{1}$, Sundol Kim² ${ }^{2}$, Sandra Zivkovic ${ }^{2}$, Yi Ren ${ }^{2}$, Hoyong Chung ${ }^{1}$, Jingjiao Guan ${ }^{1}$ \\ ${ }^{1}$ Department of Chemical and Biomedical Engineering, FAMU-FSU College of Engineering, \\ Tallahassee, Florida 32310, USA \\ guan@eng.famu.fsu.edu \\ ${ }^{2}$ College of Medicine, Florida State University, Tallahassee, Florida 32306, USA
}

\section{Extended Abstract}

Phagocytosis is a process by which a cell such as macrophage internalizes a micrometre-sized particle. It plays critical roles in immunity, tissue remodelling and repair.[1] Phagocytosis is characterized by the internalization of the particle in a vacuole called phagosome inside the cell. Formation, maturation and resolution of the phagosome is a complex process and is not well understood. Among this process is the appearance and trafficking of intracellular vesicles that are apparently derived from the phagosome and named phagosome-derived vesicles (PDVs). Existing techniques for tracking PDVs rely on using particles that can be enzymatically degraded into soluble fluorescent fragments inside the phagosome. These techniques are incapable of detecting whether components of the phagosome membrane are in the PDVs and tracking PDVs formed from phagosomes containing non-degradable particles. The objective of this study is to develop a novel method for tracking PDVs derived from membranous components phagosomes containing non-degradable particles. The method is featured by using microparticles fabricated with a microfabrication technique. The technique, which is based on integrating spin-coating and microcontact printing, allows a precise control on the size and composition of the microparticles.[2] The microparticles are loaded with a dialkylcarbocyanine dye for staining the membrane of the PDVs. In this study, we have established a protocol for fabricating the microparticles with a temperature-sensitive polymer. The microparticles can be readily phagocytosed by a commonly used model macrophage cell line. By using a fluorescent-temperature sensitive polymer, we have confirmed that the dialkylcarbocyanine dye can stain intracellular vesicles outside the phagosomes while the phagosomes maintain their membrane integrity. Moreover, most of the dye-stained vesicles are formed after the microparticles have been phagocytosed, indicating that the dye-stained vesicles are PDVs. Additionally, we have made the following findings about the dye-stained vesicles. (1) They can move longitudinally along the pseudopodia of the macrophages and tend to accumulate in the pseudopodia. (2) They are acidic as revealed by Lysoview staining. (3) They are co-localized with vesicles stained by a different dialkylcarbocyanine dye added in the cell-culture medium. (4) They are colocalized with vesicles stained by a fluorescent dextran added into the cell-culture medium. (5) They are colocalized with zymosan bioparticles that are later phagocytosed by the macrophages. These findings indicate that the PDVs can be formed from phagosome containing non-degradable particles; the PDVs contain membranous components of the phagosomes; and the PDVs allow material transfer between phagosomes within a macrophage. In addition to the model macrophages, this technique can, in principle, be used to track PDVs in other types of phagocytes such as microglia, which play a critical role in the pathology of Alzheimer's disease.

\section{References}

[1] A. Aderem and D.M. Underhill, "Mechanisms of phagocytosis in macrophages", Annu Rev Immunol. Vol. 17, pp. 593623, 1999

[2] Guan J., Ferrell N., Lee L.J., Hansford D.J., "Fabrication of polymeric microparticles for drug delivery by soft lithography", Biomaterials Vol. 27 (21), pp. 4034-4041, 2006 